\section{Dr. de Rotte, et al reply}

\section{To the Editor:}

We thank Dr. Ranganathan for her interesting comments ${ }^{1}$ on our work ${ }^{2}$. In our recently published prediction model for methotrexate (MTX) nonresponse in juvenile idiopathic arthritis (JIA) ${ }^{3}, A B C B 1$ rs1045642 was described, indicating the relative importance of this polymorphism to predict nonresponse to MTX in JIA. Also, we were able to reproduce this finding in a prospective cohort of 387 adult patients with rheumatoid arthritis (RA) receiving MTX: the $A B C B 1$ rs1045642 polymorphism showed an association with improved clinical response (lower Disease Activity Score-28; $\beta=-0.16, p=0.001$ ). We agree that finding genetic predictors for MTX-induced toxicity and gastrointestinal (GI) adverse events is equally as important as response, because toxicity limits the considerations of a dose increase or continuation of MTX, and GI adverse events could result in overt refusal by children with JIA to take MTX, making an alternative therapy more appropriate.

In JIA, effects of toxicity such as bone marrow suppression and elevated liver enzymes occur rarely, leading to a lack of power to perform pharmacogenetic testing. In contrast, GI adverse events occur frequently ${ }^{4}$. We recently developed and validated a questionnaire for GI adverse events in patients with JIA ${ }^{4}$. Using this questionnaire, we documented GI adverse events such as abdominal pain, nausea, and vomiting as well as fatigue in a prospective cohort of 140 patients with JIA. We then tested associations of GI adverse events with polymorphisms in MTX transporter genes ${ }^{2}$, in particular 3 months after start of MTX, because GI adverse events shortly after starting MTX have the most influence on limiting dose increases or continuation. Associations were tested with a univariate logistic regression analysis.

Within 3 months of starting MTX, $46 \%$ of the patients reported abdominal pain, $43 \%$ nausea, $11 \%$ vomiting, and $49 \%$ fatigue. There was a trend toward associations of $A B C C 2$ rs 4148396 (OR 0.52, 95\% CI $0.26-1.05, \mathrm{p}=0.070)$ and $A B C C 3 \mathrm{rs} 4793665$ (OR 0.49, 95\% CI 0.24-1.01, $\mathrm{p}=0.052$ ) polymorphisms with nausea. The SLC19A1 rs1051266 polymorphism showed a trend to association with abdominal pain (OR 2.76, $95 \%$ CI $0.88-8.62, \mathrm{p}=0.081$ ). The $A B C C 3$ rs 4793665 (OR $0.33,95 \%$ CI $0.12-0.91, \mathrm{p}=0.031$ ) and SLC19A1 rs1051266 (OR 2.94, 95\% CI $1.37-6.31, \mathrm{p}=0.006$ ) polymorphisms were associated with fatigue. For these findings to be useful in daily clinical practice, multivariate analyses, metaanalyses, and validation studies are needed, eventually leading to construction of prediction models. We are currently constructing a prediction model for GI intolerance, as we did for MTX nonresponse in $\mathrm{JIA}^{3}$.

As Dr. Ranganathan stated, it would be interesting to know whether MTX pharmacogenetic associations are comparable between children with JIA and adults with RA. Therefore, we also have investigated transporter gene polymorphisms in a cohort of 387 adult patients with RA. The following adverse events were analyzed after 3 months of therapy: GI events (diarrhea, vomiting, and sickness or abdominal pain) and malaise (fatigue, dizziness, headache, and sleeplessness or feeling not well). GI adverse events were observed in $43 \%$ of the patients and malaise in $45 \%$.
In univariate logistic regression analysis, we found only 1 significant association: the $A B C B 1$ rs 1045642 polymorphism was associated with malaise (OR 2.57, 95\% CI 1.59-4.15, p < 0.001).

Hence, in our RA cohort, we did not reproduce the associations between polymorphisms and adverse events observed in our JIA cohort. These findings could indicate that the genetics/MTX outcome relations may differ between children and adults. Although we did observe effects of transporter gene polymorphisms on GI adverse events in JIA and RA, we were not able to replicate the findings of Ranganathan, $e t a l^{5}$. This underscores the need for metaanalyses and collaborations between centers to build prediction models for outcomes of MTX therapy in pediatric and adult rheumatic diseases.

MAURITS C.F.J. de ROTTE, PharmD, Department of Clinical Chemistry, Erasmus MC, University Medical Center, Rotterdam; MAJA BULATOVIC, MD; MARLOES W. HEIJSTEK, MD; NICO M. WULFFRAAT, MD, PhD, Department of Pediatric Immunology, University Medical Center Utrecht, Wilhelmina Children's Hospital, Utrecht; ROBERT de JONGE, PhD, Department of Clinical Chemistry, Erasmus MC, University Medical Center, Rotterdam, The Netherlands. Address correspondence to R. de Jonge, Department of Clinical Chemistry, Erasmus MC, 's-Gravendijkwal 230, 3015 CE, Rotterdam, The Netherlands. E-mail: r.dejonge@erasmusmc.nl

Supported by the Dutch Arthritis Association (no. 06-02-402 to R.J. and no. 07-01-402 to N.M.W.).

\section{REFERENCES}

1. Ranganathan $\mathrm{P} . \mathrm{ABC}$ transporter genes and methotrexate response in rheumatoid arthritis [letter]. J Rheumatol 2013;40:536.

2. de Rotte MC, Bulatovic M, Heijstek MW, Jansen G, Heil SG, van Schaik RH, et al. ABCB1 and ABCC3 gene polymorphisms are associated with first-year response to methotrexate in juvenile idiopathic arthritis. J Rheumatol 2012;39:2032-40.

3. Bulatovic M, Heijstek MW, Van Dijkhuizen EH, Wulffraat NM, Pluijm SM, de Jonge R. Prediction of clinical non-response to methotrexate treatment in juvenile idiopathic arthritis. Ann Rheum Dis 2012;71:1484-9.

4. Bulatovic M, Heijstek MW, Verkaaik M, van Dijkhuizen EH, Armbrust W, Hoppenreijs EP, et al. High prevalence of methotrexate intolerance in juvenile idiopathic arthritis: Development and validation of a methotrexate intolerance severity score. Arthritis Rheum 2011;63:2007-13.

5. Ranganathan P, Culverhouse R, Marsh S, Mody A, Scott-Horton TJ, Brasington R, et al. Methotrexate (MTX) pathway gene polymorphisms and their effects on MTX toxicity in Caucasian and African American patients with rheumatoid arthritis. J Rheumatol 2008;35:572-9.

J Rheumatol 2013;40:4; doi:10.3899/jrheum.121496 